\title{
Histopathological and immunohistochemical studies of a typical case of intraductal papillary carcinoma of a male breast.
}

\author{
Jaydeep Nath ${ }^{1}$, Simanti Sinha ${ }^{1}$, Abhishek Mukherjee ${ }^{1}$, Tridip Chatterjee ${ }^{2}$ \\ ${ }^{1}$ Suraksha Diagnostic Centre, Salt Lake, Kolkata, West Bengal, India. \\ ${ }^{2}$ Suraksha Genomics (R\&D Division of Suraksha Diagnostic), Salt Lake, Kolkata, West Bengal, India.
}

\begin{abstract}
Intraductal papillary carcinoma is very uncommon breast disease, constituting $0.5 \%$ to $1 \%$ of all breast cancers. Intraductal and intracystic papillary carcinomas are the histological classification of papillary carcinomas. Here we report, a 61 year old man presented with a mass in the upper outer quadrant of the left breast, with associated nipple discharge and bleeding since 2 months. Histological examination revealed tumor cells arranged in multi-cell layered papillae mostly in dilated lumen with foci of cribriform, follicular and solid pattern. Occasional psammoma bodies are also noted. Surgical excision is usually recommended after core-needle biopsy if there is atypia, high risk lesion, imaging histological discordance or positivity for malignancy. Often biopsy excision is performed directly when papillary carcinoma is suggested by sonography or mammography. Indeed, the surgical excision allows the pathologist to classify the papillary by classically histological examination of the lesions especially with immunohistochemical study and to research invasion or DCIS in surrounding breast tissue, present in majority of cases. There are no evidence based guidelines for treatment of intraductal papillary carcinoma. Hence, it is recommended to go for conservative surgery without axillary dissection, which shows excellent prognosis.
\end{abstract}

Keywords: Intraductal papillary carcinoma, Intracystic papillary carcinoma, Ductal carcinoma, In situ (DCIS).

\section{Introduction}

Breast carcinoma in men is extremely rare; it represents $0.6 \%$ of all breast carcinomas and less than $1 \%$ of all malignancies in men [1]. Intraductal papillary carcinoma (IPC) is also a rare form of breast cancer, accounting for $0.5-1 \%$ of all breast cancers [2]. IPC in man is an extremely rare disease with only very few case reports published in the literature so far [3-5]. IPC accounts for 5 to $7.5 \%$ of all male breast cancers [6]. It typically occurs at an old age with a good prognosis [7]. Intraductal papillary carcinoma is a rare form of malignant breast disease, which constitute about $0.5 \%$ to $1 \%$ of all breast cancers.

Papillary carcinomas can be classified into intraductal and intracystic papillary carcinoma as per histopathological evaluation. It has been found that the incidence and prevalence of both invasive and in situ papillary carcinoma is greater in postmenopausal elderly women, and in males. Clinically, the patient is asymptomatic or present with a breast mass with or without nipple discharge. In radiological investigations, intraductal papillary carcinoma has present mostly with benign features. Histological examination shows a well circumscribed mass lesion having proliferation of neoplastic cells arranged around fibrovascular cores. From therapeutic as well as prognostic points distinction of invasive papillary carcinoma from non-invasive forms is very important.

Papillary neoplasm of breast encompasses a heterogeneous group of lesions in histomorphology, all sharing a growth pattern characterized by the presence of fibro vascular stalks lined by neoplastic epithelial cells. Malignant papillary neoplasms of the breast consist of a number of microscopically distinct lesions including ductal carcinoma in situ (DCIS) arising in an intraductal papilloma, papillary DCIS, encapsulated papillary carcinoma, solid papillary carcinoma and invasive papillary carcinoma.

\section{Case Report}

A 61 year old man presented with a mass in the upper outer quadrant of the left breast, with associated nipple discharge and bleeding since 2 months. On physical examination one $2 \mathrm{~cm}$ diameter mass noted with ill-defined margins. The lesion is fixed and firm but painless, and without any skin changes. Mammography showed an oval opacity of the upper outer quadrant with calcifications. Ultrasonography showed a solid-cystic mass measuring $27 \mathrm{~mm}$, with posterior acoustic enhancement. FNAC had done revealed nuclear atypia. Surgical excision was carried out.

Histological examination revealed tumor cells arranged in multicell layered papillae mostly in dilated lumen with foci of cribriform, follicular and solid pattern. Occasional psammoma bodies are also noted. There is no evidence of microscopic invasion or lymphovascular invasion or involvement of any of the ten axillary nodes dissected (0/10). Nipple and areola as well as posterior resection margin are not involved (Figures 1 and 2). 


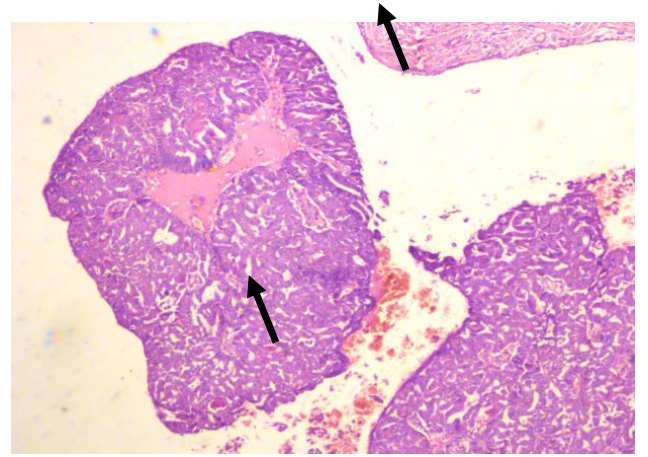

100X: Papillary fragments with fibrovascular core (H\&E stain)

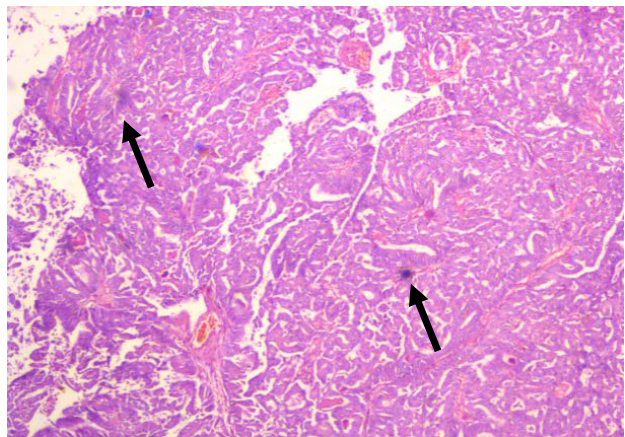

100X: Papillary fronds with psammoma bodies (H\&E stain)

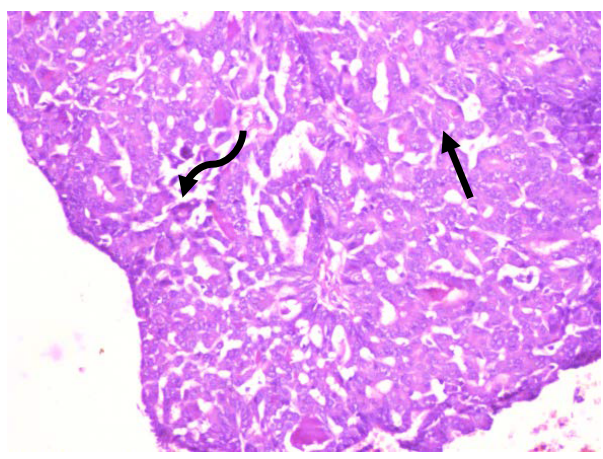

400X: Papillary strands with vesicular nuclei (straight arrow) and psammoma bodies (curved arrow) [H\&E stain].

Figure 1. Histological examination revealed tumor cells arranged in multi-cell layered papillae.

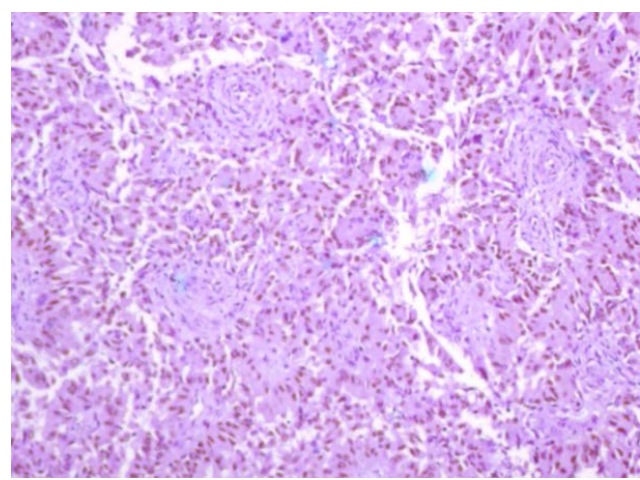

400X: ER positive staining of tumor cells

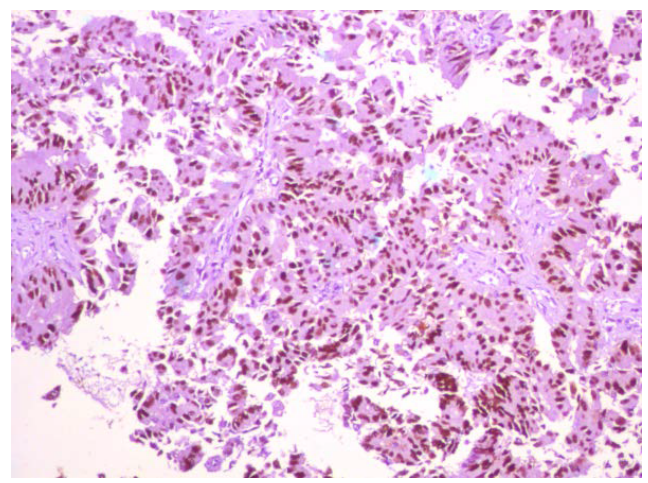

400X: PR positive nuclei of the papillary cores

Figure 2. Immunohistochemical findings reveal strong positivity for ER and PR. Allred scoring show high intensity (3) and proportion (5) score for both ER and PR.

\section{Discussion}

Papillary carcinoma of the breast is a rare form of malignant tumor, constituting $1-2 \%$ of all breast carcinomas in women. It is characterized by the papillary architecture with proliferation of neoplastic cells forming finger-like projections or fronds having central fibrovascular cores covered by epithelium without myoepithelial cell layer. Presence of myoepithelial cells differentiate between benign and malignant papillary lesion. Lesions can be divided into invasive and non-invasive forms. Non-invasive papillary carcinomas are subdivided further into two subtypes, a diffuse form the papillary variant of ductal carcinoma in situ and a localized formintracystic papillary carcinoma. In mammography, the intraductal papillary carcinoma is seen as a round to oval or lobulated opacity. The margins of the mass lesions are usually circumscribed but in some cases indistinct or obscured margins are also found in places indicating presence of inflammation or invasion. The co-existence of a residual palpable mass with a frankly bloody aspirate at the fine needle aspiration cytology is a strong indicator of carcinoma.

Many authors use core needle biopsy of the intraductal mass under ultrasonographic guidance as a distinguishing tool between benign from malignant papillary lesions, but it has got a low accuracy for identifying in situ or invasive papillary carcinoma because the site of biopsy is generally central where the invasion is usually found at the periphery of the tumor mass.

Surgical excision is the standard recommendation after core-needle biopsy if there is atypia, any high-risk lesion, positivity for malignancy, or imaging-histological discordance. Excision biopsy is often performed, when papillary carcinoma is suggested by mammography or sonography. Surgical excision allows the pathologist to classify the papillary lesions by conventional histological examination aided with immunohistochemical study and to demonstrate invasion or DCIS in surrounding breast tissue, present in majority of cases. 
There are no strict evidence based guidelines for treatment of intraductal papillary carcinoma. Randomized controlled trials comparing outcome of breast conserving surgery to mastectomy is also not very promising. There are also many case reports as well as retrospective studies that showed excellent prognosis with conservative surgery without dissection at axillary part. In this context, we believe this paper will enlighten all concern about the extremely rare Intraductal Papillary Carcinoma of a male breast.

\section{Conflict of Interest}

The authors declare no conflict of interest.

\section{References}

1. Anderson WF, Devesa SS. In situ male breast carcinoma in the surveillance, epidemiology, and end results database of the national cancer institute. Cancer 2005; 104: 1733-1741.

2. Solorzano CC, Middleton LP, Hunt KK, et al. Treatment and outcome of patients with intracystic papillary carcinoma of the breast. Am J Surg 2002; 184: 364-368.
3. Lam WWM, Tang APY, Tse G, et al. Radiology-pathology conference: Papillary carcinoma of the breast. Clin Imaging 2005; 29; 396-400.

4. Rosen PP. Papillary carcinoma; Rosen's Breast Pathology. Lippincott-Ravel Publishers, Philadelphia, Pa, USA. 1997; 335-354.

5. Muttarak M, Samwangprasert A, Chaiwun B. Intracystic papillary carcinoma of the breast. Biomedical Imaging and Intervention Journal 2005; 1: 52.

6. Sinha S, Hughes RG, Ryley NG. Papillary carcinoma in a male breast cyst: A diagnostic challenge. Ann R Coll Surg Engl 2006; 88: W3-W5.

7. Romics L, O'Brien ME, Relihan N, et al. Intracystic papillary carcinoma in a male as a rare presentation of breast cancer: A case report and literature review. J Med Case Rep 2009; 3: 13 .

\section{Correspondence to:}

Tridip Chatterjee

Suraksha Genomics (R \& D Division of Suraksha Diagnostic)

DD-18/1. Sector 1, Salt Lake, Kolkata

West Bengal,

India.

Tel: +91-98313252580,

E-mail: ctridip@gmail.com, tridip.academic@gmail.com 